\title{
Subsample difference correction for Terra MODIS SWIR bands 5-7 using lunar observations
}

\author{
Truman Wilson ${ }^{a}$ and Xiaoxiong Xiong ${ }^{b}$ \\ ${ }^{a}$ Science Systems and Applications, Inc., 10210 Greenbelt Road, Lanham, MD 20706, USA; \\ ${ }^{b}$ Sciences and Exploration Directorate, NASA/GSFC, Greenbelt, MD 20771, USA
}

\begin{abstract}
The Moderate Resolution Imaging Spectroradiometer (MODIS) is one of the key instruments on board the Terra (EOS AM-1) spacecraft. MODIS has 36 spectral bands ranging in wavelength between 0.4 and $14.2 \mu \mathrm{m}$, at three spatial resolutions of $250 \mathrm{~m}$ (bands 1-2), $500 \mathrm{~m}$ (bands $3-7$ ), and $1 \mathrm{~km}$ (bands $8-36$ ). For each 1-km sample, the 250-m and 500-m bands use 4 and 2 detectors with each acquiring 4 and 2 subsamples respectively in order to maintain consistent along-scan and along-track resolutions at nadir. The SWIR bands, $5-7$ and 26 , share the same focal-plane array as the 1-km thermal emissive bands, $20-25$. During one of the two 500-m subsamples for bands $5-7$, sampling of the 1-km bands introduces increased electronic crosstalk contamination, resulting in a subsample difference for both Earth-view and on-board calibrator observations. For this work, we use data from lunar and on-board blackbody observations, which occur at different signal levels for bands $20-25$, to derive a correction to the contamination. This correction can be applied to reduce the subsample differences in the MODIS Earth-view data over a wide range of scenes. The impact of this correction on the sensor calibration and Earth-view data will be assessed.
\end{abstract}

Keywords: MODIS, Terra, SWIR, electronic crosstalk, reflective solar bands

\section{INTRODUCTION}

The Moderate Resolution Imaging Spectroradiometer (MODIS) has been in operation on board the Terra spacecraft since its launch on December 18, 1999. ${ }^{1}$ MODIS is a whisk-broom, scanning radiometer with 36 separate spectral bands which range in wavelength from $0.4-14.2 \mu \mathrm{m}$. These bands are grouped by wavelength into the reflective solar bands (RSB, bands $1-19$ and $26, \lambda<2.1 \mu \mathrm{m}$ ) and thermal emissive bands (TEB, bands $20-25$ and $27-36, \lambda>3.6 \mu \mathrm{m}$ ). Each of the TEBs has a 1-km spatial resolution at nadir, and the RSBs are further divided into 3 nadir resolutions with bands $1-2$ at $250 \mathrm{~m}$, bands $3-7$ at $500 \mathrm{~m}$, and bands $8-19$ and 26 at $1 \mathrm{~km}$. The MODIS bands consist of linear arrays of detectors, with 40, 20, and 10 detectors for the 250-m, 500-m, and $1-\mathrm{km}$ bands respectively. Each of the bands is located on one of four focal plane assemblies (FPA) which group the bands by wavelength: the visible (VIS), near-infrared (NIR), short- and mid-wave infrared (SWIR/MWIR), and long-wave infrared (LWIR). For each MODIS scan, every band acquires an Earth-view (EV) image that is $10 \mathrm{~km}$ in the track direction at nadir and $2330 \mathrm{~km}$ in the scan direction. Each scan is divided into 1354 samples (frames) per band, with the higher resolution bands acquiring additional subsamples (subframes) in order to maintain an equal resolution in the scan and track directions at nadir.

Each of the MODIS bands is calibrated on orbit in order to track the gain degradation of the individual detectors. The on-board calibrators (OBC) of MODIS include a solar diffuser (SD) with its corresponding SD stability monitor (SDSM) for calibrating the RSBs, ${ }^{2}$ and a blackbody (BB) for calibrating the TEBs, ${ }^{3}$ as seen in Figure 1. Additional spatial, spectral, and radiometric characterizations can be performed using the spectroradiometric calibration assembly (SRCA). ${ }^{4,5}$ During each MODIS scan, the scan-mirror will view each of the OBCs in succession along with an EV acquisition. MODIS also uses observations of the Moon through the space-view (SV) port in order to characterize the scan-mirror response versus scan angle (RVS) at a different angle-of-incidence than the SD for the RSBs. ${ }^{2}$ Observations of selected EV targets are also used for additional characterizations of the RVS for the RSBs. ${ }^{6,7}$

Further author information: (Send correspondence to T.W.)

T.W.: E-mail: truman.wilson@ssaihq.com, Telephone: 13018672120 

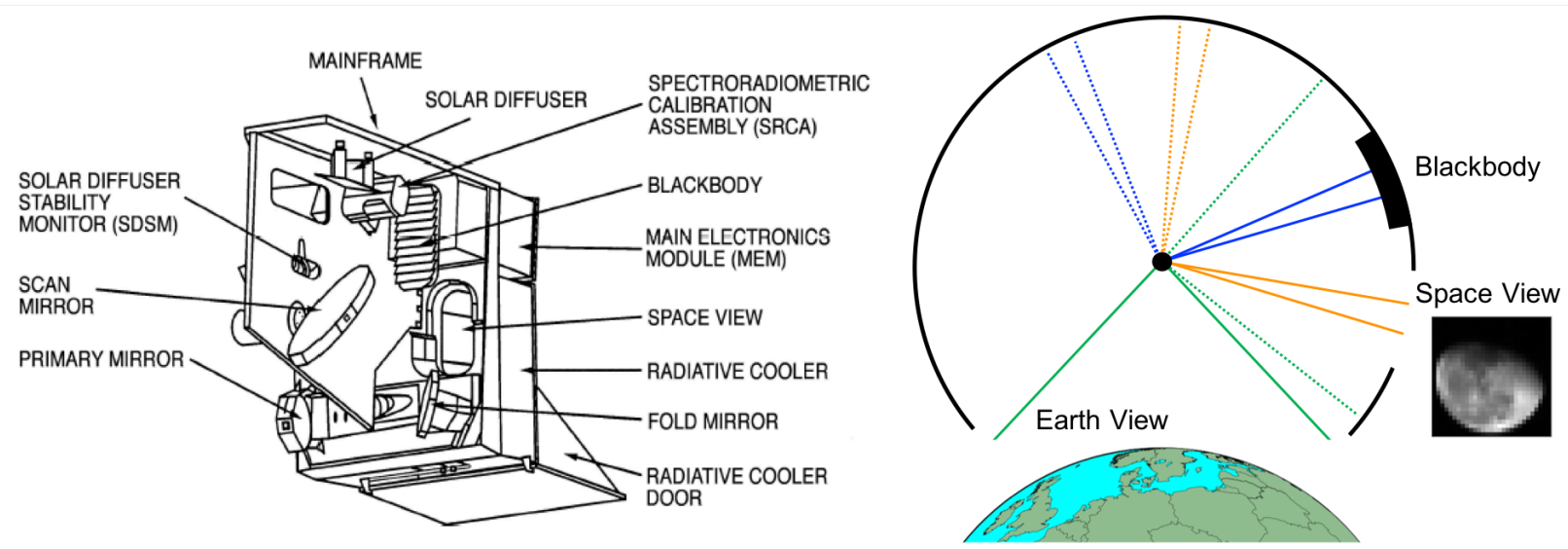

Figure 1. (Left) A diagram of the MODIS layout including the OBCs and SV port. (Right) A diagram showing the angular views of the $\mathrm{EV}, \mathrm{SV}$, and $\mathrm{BB}$ data sectors during normal operation (solid lines) and during the sector rotation (dotted lines). This diagram is illustrative, and is not shown to scale. Data sectors for the other OBCs are also not shown.

For Terra MODIS, electronic crosstalk between the bands has been observed since the mission beginning for the MODIS SWIR bands ${ }^{8}$ the LWIR bands, ${ }^{9,10}$ the MWIR bands, ${ }^{11,12}$ and band $2 .{ }^{13}$ For the SWIR bands, a correction to the crosstalk has been implemented in the MODIS Level-1B (L1B) data since Collection 2 in 2000. In recent years, the issue has had a particularly significant impact for the LWIR bands $27-30$. A correction to these bands has been applied to the MODIS L1B throughout the entire mission in Collection 6.1. ${ }^{14}$ For the TEBs, lunar observations were used in order to characterize the contamination and derive correction coefficients which could be applied to the MODIS data.

The SWIR bands in MODIS also have signal contamination in the form of an out-of-band optical leak (OL). This OL is at a wavelength of $5.3 \mu \mathrm{m}$, which is in the wavelength range of the TEBs, as seen in Figure 2 for bands $5-7$. Because of this, these bands measure a non-background level signal during nighttime scenes and when viewing the on-board BB (Figure 2, right). To make an effective correction for both the OL and electronic crosstalk, the current L1B data uses a correction derived from nighttime scenes where data from the RSBs are stored, which is typically done only during daytime scenes, and is known as nighttime day-mode (NTDM). ${ }^{8}$ The correlation between the SWIR signal and a reference band (28 for Terra, 25 for Aqua) is then used to derive
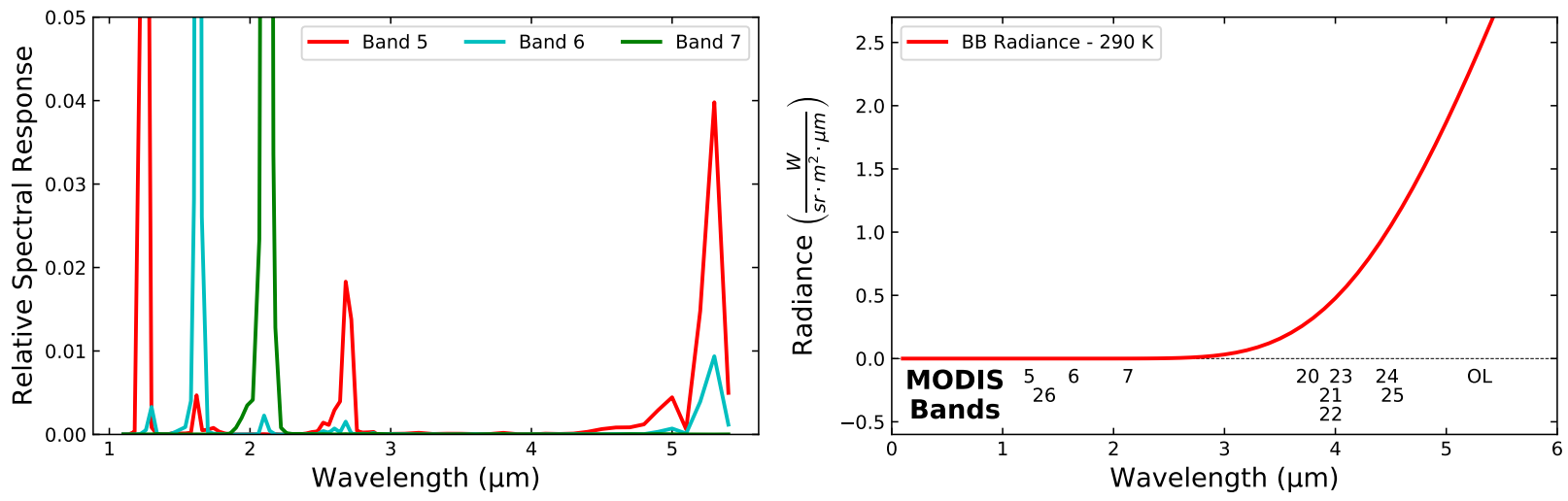

Figure 2. (Left) Relative spectral response for the 500-m SWIR bands $(5-7)$ in Terra MODIS. An out-of-band OL can be seen as a peak at $5.3 \mu \mathrm{m}$. For band 7, the OL is much lower than what is measured for bands 5 and 6 in the spectral response data. (Right) The radiance for the on-board BB as a function of wavelength at $290 \mathrm{~K}$. The wavelength of the MODIS SWIR and MWIR bands are indicated by labels at the appropriate wavelength. The wavelength of the optical leak is also shown. 
correction coefficients which are applied to SWIR data. While this NTDM-based OL correction reduces the level of signal contamination, for bands $5-7$ there is still a difference in the subsample retrieval that can be observed, as we will show in Section 3.

Since the OL though the SWIR band filters is, in principle, independent of the subsample retrieval, the difference that remains is likely due to the electronic component of the contamination that is not fully accounted for in the NTDM-based correction. In Figure 3, we show a diagram of the electronics sampling for the 500-m and $1-\mathrm{km}$ bands on the SWIR/MWIR FPA. The 500-m and 1-km bands are sampled on different electronic outputs. For each MODIS frame, the detectors for a given set of bands are sampled in order in a repeating cycle on their respective electronic outputs. Since the 500-m bands sample twice as often as the 1-km bands, for one subframe of 500-m band sampling, the 1-km bands are not used, while for the other subframe they are sampled simultaneously. In previous work, when using lunar observations to look for signs of electronic crosstalk, there was a clear subframe difference in the level of contamination, ${ }^{15}$ as seen in Figure 3. For bands 5 and 7 , contamination from the 1-km TEBs on the same FPA only shows signs of sending contamination in subframe 2. For band 6, since it is offset by a half frame on the FPA (and is shifted when the data is sent down from the spacecraft), this contamination is only seen in subframe 1. For simplicity, in this work the subframes will be referred to in terms of the contamination seen in bands 5 and 7 , with the assumption that band 6 has the reversed effect. It should be noted that before September 2001, the electronics configuration of the instrument was changed multiple times in order to minimize the effects of electronic crosstalk, which showed good results, particularly for subframe 1 as seen in the lunar data. During the early part of the mission, some of the electronics configurations showed positive contamination for both subframes in the lunar data. This early mission behavior is difficult to characterize and will be the subject of future work.

The subframe difference is also observed when viewing the BB with the SWIR bands, as shown in Figure 3 for band 5. For subframe 1, the background-subtracted signal is positive, which is consistent with contamination from the OL. However, for subframe 2, the signal level is negative, which is a strong indication of electronic crosstalk. These observations match what we see in the lunar data. While the dominant component of the

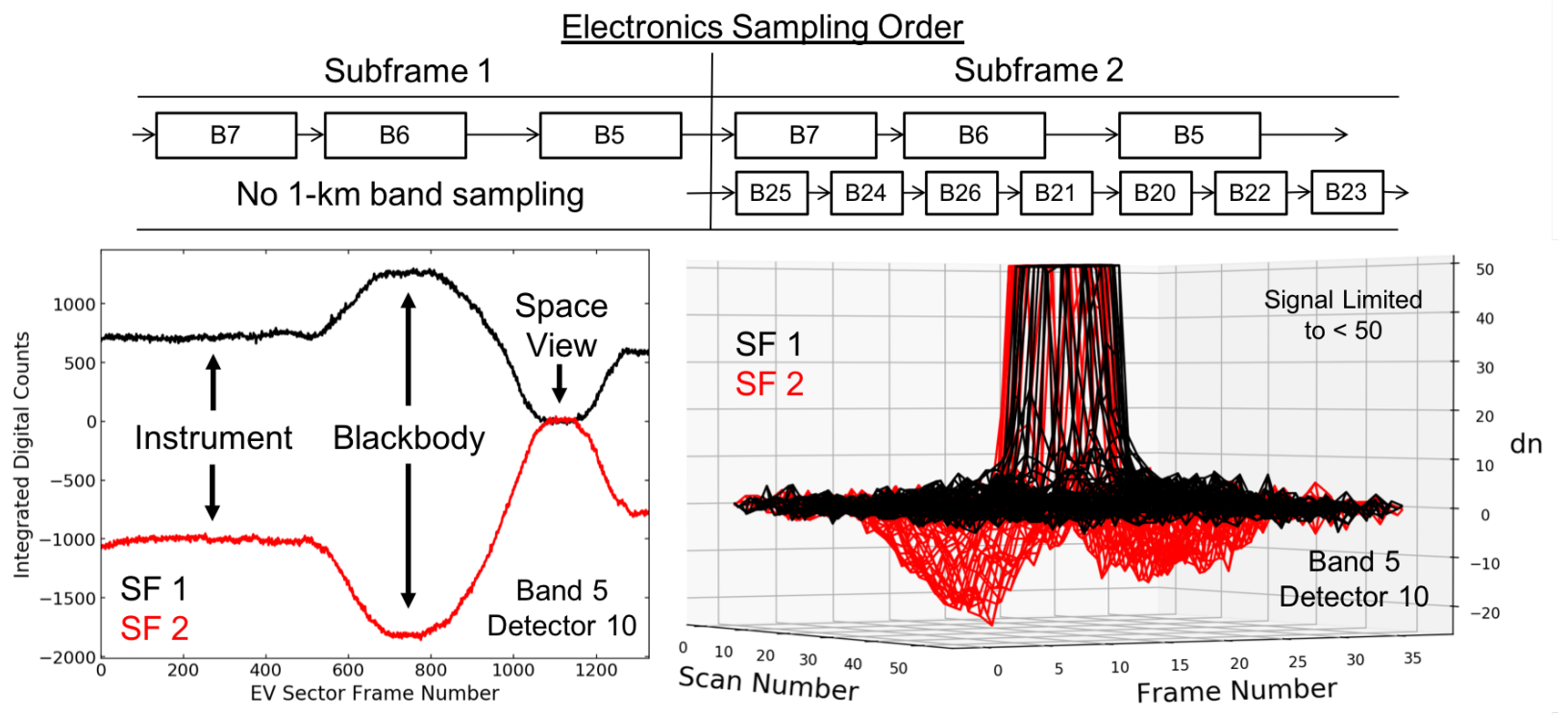

Figure 3. (Top) Diagram of the electronics sampling order for the SWIR and MWIR bands. In the first subframe, there is only sampling of the 500-m bands. In the second subframe, the $1-\mathrm{km}$ bands are sampled with the 500-m bands at the same time on a separate electronic output. (Bottom Left) An example of the sector-rotated data without the Moon present for band 5 detector 10, with data from both subframes shown. The background level is set to the level of the SV data. (Bottom Right) An example of the lunar data plotted in 3 dimensions. For subframe 2 in both of the bottom charts, a negative signal with respect to the space-view signal level indicates the presence of electronic crosstalk from the 1 -km bands. 
contamination in the second subframe is from electronic crosstalk, it is expected to have a similar level of contamination from the OL as the first subframe.

In previous work, we derived a correction to the electronic crosstalk from lunar observations similar to our TEB correction for the SWIR bands and applied it to the lunar calibration results. ${ }^{15}$ While this correction resulted in a reduction in the oscillations in the lunar calibration, application of the coefficients to the EV data was insufficient for removing the subsample differences in the L1B. Unlike the TEB electronic crosstalk correction, the SWIR bands receive contamination from a different electronic output on the FPA from the 1-km bands. Because of this, it may be the case that the large levels of saturation seen for the TEBs when viewing the Moon play a role in artificially reducing the correction coefficient values before they can be applied to the EV data.

In this work, we will introduce a new method for deriving crosstalk correction coefficients for bands $5-7$ that will use data from scheduled lunar observations. In Section 2, we will discuss the MODIS lunar data and the development of the algorithm. We will take advantage of the data sector rotation during the lunar observations that allows us to view the Moon, BB, and the inside of the instrument simultaneously. Since the $\mathrm{BB}$ and instrument data is at a level that does not saturate the sending bands, we will use it as the reference for the correction, while obtaining additional information about the sending bands from the lunar data. From this data, we will derive correction coefficients for each band, detector, and subframe individually. In Section 3, we will discuss the impact of the correction on the L1B data and assess the ability of the coefficients to remove the subsample difference observed for each band. Here, we will assess the impact of the correction over a large range of radiance levels and on the L1B imagery. Finally, we will discuss potential improvements to the correction and future work.

\section{MODIS LUNAR OBSERVATIONS AND ALGORITHM DEVELOPMENT}

Both Terra and Aqua MODIS use lunar observations in order to characterize the change in the scan-mirror RVS relative to the SD. ${ }^{16}$ These observations have occurred on a near monthly basis throughout the entire mission and use a spacecraft roll maneuver in order to bring the Moon into alignment with the SV port at the appropriate lunar phase angle. ${ }^{17}$ While the primary purpose of the lunar observations is for radiometric calibration, they have also been used extensively for the characterization of electronic crosstalk in the MODIS bands ${ }^{10-12,14,15}$ and for spatial characterization. ${ }^{18,19}$ For Terra MODIS bands $27-30$, a correction to electronic crosstalk derived from lunar observations has been implemented in MODIS Collection 6.1. ${ }^{14}$

The MODIS SV port is used to obtain the background signal level for the MODIS bands relative to the other data sectors (EV, SD, BB, etc.). During normal operation, MODIS obtains data from a portion of the SV port, 50 frames $\left(\sim 4^{\circ}\right)$, whereas the total SV port width is approximately 75 frames $\left(\sim 6^{\circ}\right)$. The EV data sector is 1354 frames and subtends an angle of approximately $110^{\circ}$. In order to ensure that the Moon is observed during the spacecraft maneuver, we use a data sector rotation which rotates the EV sector so that it views both the SV port and the BB, as seen in Figure 1. This sector rotation moves each data sector view off of its normal target during the data collection.

Since the Moon is further away from the sensor than the Earth, MODIS is able to view the entire disk of the Moon in a single scan, as it is approximately the width of $6-7$ MODIS 1-km pixels. Also, the Moon appears in the image over many successive scans, as it moves across the FPA by less than a single 1-km MODIS pixel from scan-to-scan. This effect is known as oversampling, ${ }^{16}$ and it allows us to create images of the Moon using data from only a single detector.

The Terra MODIS lunar observations have a data collect time of 3 minutes and 30 seconds which coincides with the sector rotation. For a fraction of the scans a partial or full disk lunar image is present, while for the rest of the scans, the Moon is not observed. For this work, we will find it useful to split the data into two categories where the Moon is either in (partial or full) or out of the SV port. For the scans where no Moon is present, our sector rotated data observes the SV, BB and the instrument as seen in Figure 3 (lower left). This data changes little from scan-to-scan, therefore, we represent this data using the integrated signal from each scan where the Moon is not present. For the scans where the Moon is present, it is better to represent the data in 3 dimensions over scan and frame number as seen in Figure 3 (lower right). This data is subset so that it only contains data 
from the SV port. For this data in particular, we are only interested in the low signal level behavior outside of the main lunar peak which is affected by electronic crosstalk.

For each data type, there is evidence of signal contamination due to electronic crosstalk and/or the out-ofband OL. For the BB data, we expect the signal level for the SWIR bands to be near the background level since the $\mathrm{BB}$ radiance is expected to be near-zero for these bands compared to the TEBs, as seen in Figure 2 (right). The positive signal from subframe 1 in Figure 3 is likely due to the OL, since no contamination can be seen in the lunar data. For subframe 2, the contamination is negative and contains a combination of the OL and electronic crosstalk. For the lunar data, the signal outside of the main lunar peak should be at the background level as well, but instead shows some signal below the background level outside of the main signal, as seen in Figure 3 (lower right). All of the contamination observed outside of the main signal in the lunar data is due to electronic crosstalk, since the OL contamination will be present at the same location as the main lunar signal.

To correct the OL in this work, we use the BB and instrument data from the subframe 1 data instead of the NTDM-based correction that is applied in the L1B data. We will also use band 25 instead of band 28 as a reference signal in order remove the effects of electronic crosstalk that exist in band 28 for Terra MODIS. ${ }^{8}$ The OL correction coefficient for the $i$ th detector is found through a simple minimization of $\eta^{2}$ for the BB and instrument data without the Moon as follows:

$$
\eta_{i}^{2}=\sum_{F}\left(\sum_{S} d n_{i}^{*}(S, F, S F)-C_{i, \mathrm{OL}} \sum_{S} d n_{25}\left(S, F+\Delta F_{\mathrm{OL}}\right)\right)^{2}
$$

Here, $S, F$, and $S F$ represent the scan, frame, and subframe respectively, $d n^{*}$ is the background subtracted digital counts before the correction is applied, $C_{i, \mathrm{OL}}$ is the optical leak coefficient, and $\Delta F_{\mathrm{OL}}$ is the frame offset used to align the band 25 signal to the SWIR band data. For this data, we integrate the signal over each scan where the Moon is out of the SV port. An example of the subframe 1 data before and after the OL correction for band 5 detector 10 can be seen in Figure 4. Since the OL contamination cannot currently be separated from the electronic crosstalk contamination, we estimate the impact of the OL on subframe 2 by using the coefficients from subframe 1. Since the subframe difference should be associated with electronics only and is not optical, the OL contribution should be the same for both subframes.

For subframe 2, we calculate the electronic crosstalk coefficients first by using the lunar data. Similar to previous work, ${ }^{14}$ we can use the known frame offsets of each of the sending bands on the FPA to derive the coefficients for the $i$ th detector by performing a minimization to the following equation, similar to the OL correction:
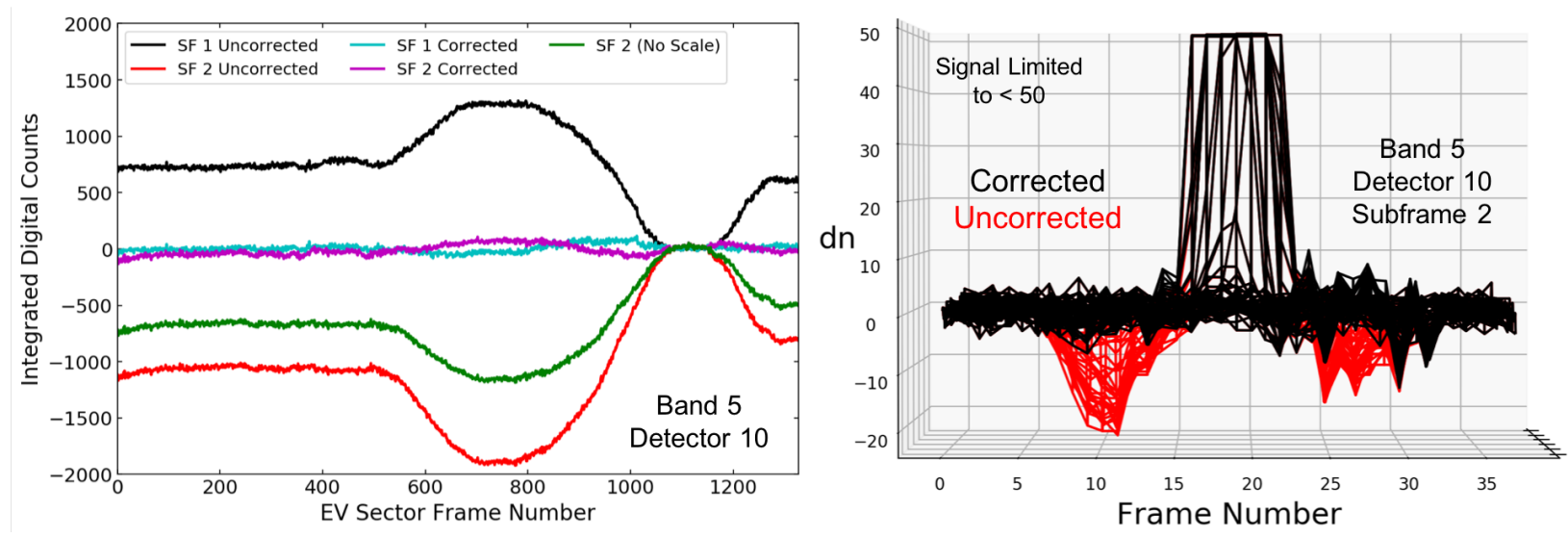

Figure 4. (Left) Example correction to the BB and instrument data using the derived coefficients that will be applied to EV data. (Right) Example lunar data correction using the saturated bands scale factor. 


$$
\eta_{i}^{2}=\sum_{S, F}\left(d n_{i}^{*}(S, F, S F)-\sum_{j} c_{i, j}(S F) \cdot d n_{j}\left(S, F+\Delta F_{j}\right)\right)
$$

Here, $c_{i, j}$ is the crosstalk coefficient matrix and $d n_{j}$ represents the sending signal from detector $j$, which in this work will consist of bands $20-26$ sending. In previous work, a small component from band $5-7$ sending was found to be negligible, and therefore it will be ignored in this work. ${ }^{15}$ Also, for this work, we use the location of the lunar contamination to further restrict the number of sending bands used to be only what we can directly correlate to a specific sending band. For bands 5 and 6, this restriction limits the sending bands to 22 and $24-26$. The coefficient values for bands 20,21 and 23 are set to zero. In the case of band 21 , which has a small signal when viewing the Moon, its inclusion can cause many issues in the later EV correction. This is because the coefficient values are difficult to derive due to the presence of neighboring bands at a much larger signal, in particular, band 22. An example of the lunar data correction for band 5 detector 10 can be seen in Figure 4 (right). For band 7, the contamination in the lunar data is not as clear as what is seen in bands 5 and 6 , and any attempts to do a sending band restriction for this band will have to be explored in the future.

If we take the coefficients derived from the lunar data and apply it to the BB and instrument data, we find that the correction is too small to bring this data back to the background level, as seen in Figure 4 (left, green). Since the EV data is closer in signal level to that of the BB and instrument as opposed to the Moon, an under correction for this data is expected to result in a corresponding under correction of the EV data, and is what we observed in our previous work. ${ }^{15}$ So, in order to provide the appropriate correction, we rescale the coefficients values of the bands that saturate (all but bands 21 and 26), keeping their relative ratios fixed, until we minimize the $\mathrm{BB}$ and instrument data, as seen in Figure 4 (left, cyan). The scaling factor applied to these coefficients typically falls within the range of $2-4$, which would indicate that the saturation plays a significant role in reducing the coefficient values.

We can use each lunar observation since September 2001 to derive correction coefficients. Before September 2001, several electronics configuration changes alter the nature of the contamination, making it difficult to develop an algorithm that can handle all of the different cases. Examples of the coefficient values for selected detectors in bands 5 and 6 are shown in Figure 5. As stated previously, the coefficient values for bands 20, 21 and 23 are fixed to zero for these bands, and therefore are not shown in these plots. In general, the coefficient values are relatively stable over the mission, with a slight change seen for the band 26 coefficient value around 2013 . In general, the band 26 sending signal is low for the lunar data, so the coefficient values derived for this band are
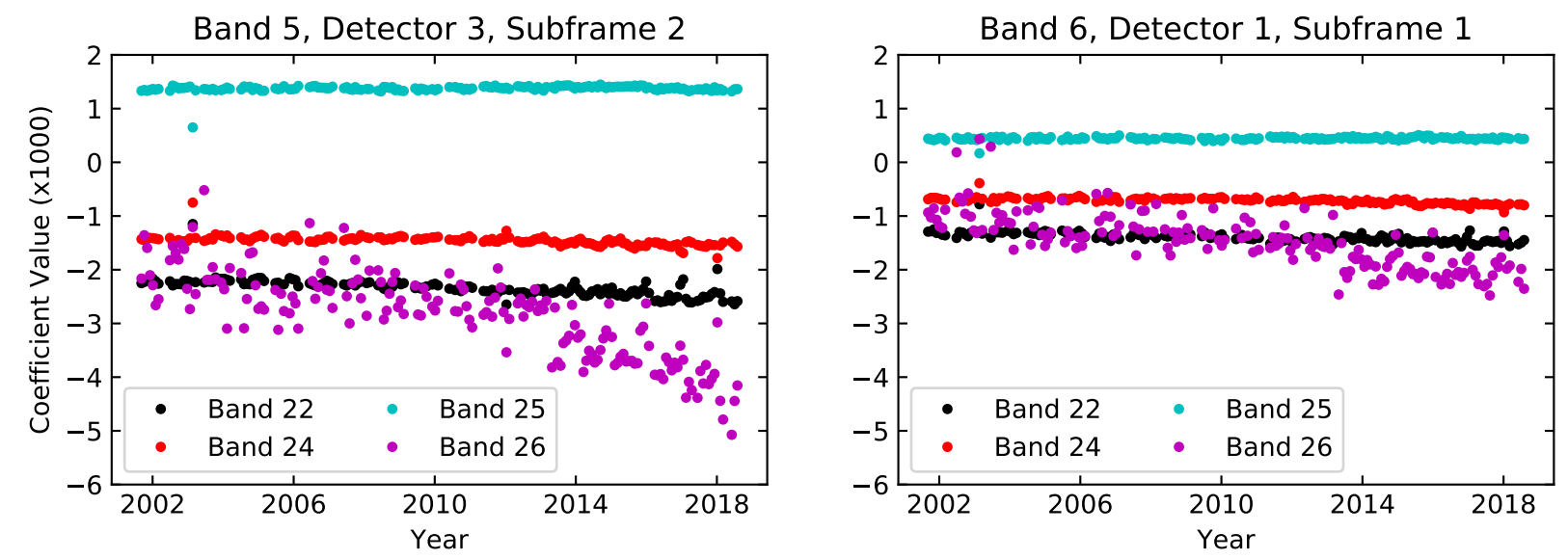

Figure 5. (Left) Example correction coefficient values for band 5, detector 3, subframe 2. (Right) Example correction coefficient values for band 6 , detector 1 , subframe 1 . The sending bands are listed in the figure legends. In each case, the band 25 coefficient values include the value of the OL coefficient. The coefficient values for bands 20,21 , and 23 are set to zero and are not shown here. 
noisier than what is derived for the TEBs sending. The coefficient values for band 5 are, in general, higher than what is measured for band 6 , including the OL term as expected. The coefficient values derived for band 7 tend to be noisy due to the band 7 lunar contamination not having as clear of an alignment between the contamination and the sending bands.

With the coefficient values, we can then apply the coefficients to the data using the following equation:

$$
d n_{i}(S, F, S F)=d n_{i}^{*}(S, F, S F)-\sum_{j} c_{i, j}(S F) \cdot d n_{j}\left(S, F+\Delta F_{j}\right)
$$

For the current formulation, the value of the OL correction coefficient is added to the electronic crosstalk coefficient values for band 25 sending. While this introduces a frame offset to the OL correction, for the large scale analysis we will be performing in this work, these offsets should have a negligible impact. This correction will be applied to both the SD calibration and to the EV data on the pixel level using Equation 3. Details of the SD calibration can be found in Reference 2. For this work, we will focus on the impact of the correction on the EV data as discussed in the following section.

\section{CORRECTION TO THE LEVEL-1B DATA}

The MODIS EV-scene radiance is calculated using the following equation:

$$
L_{\mathrm{EV}}=\frac{E_{\mathrm{sun}}}{\pi} \cdot m_{1} \cdot d n_{\mathrm{EV}}^{*}
$$

Here, $E_{\mathrm{sun}}$ is the solar irradiance, $m_{1}$ is the calibration coefficient, and $d n_{\mathrm{EV}}^{*}$ is the temperature-corrected and background-subtracted EV signal level. The calibration coefficient, $m_{1}$, is computed on orbit using the SD and the following equation:

$$
m_{1}=\frac{\rho_{\mathrm{SD}} \cdot \cos \left(\theta_{\mathrm{SD}}\right)}{d n_{\mathrm{SD}}^{*} \cdot d_{\mathrm{ES}}^{2}} \cdot \Gamma_{\mathrm{SDS}} \cdot \Delta_{\mathrm{SD}}
$$

where $\rho_{\mathrm{SD}}$ is the pre-launch bi-directional reflectance factor of the $\mathrm{SD}, \theta_{\mathrm{SD}}$ is the angle of illumination from the sun on the $\mathrm{SD}, d n_{\mathrm{SD}}^{*}$ is the temperature-corrected and background-subtracted digital signal of the $\mathrm{SD}, d_{\mathrm{ES}}$ is the Earth-Sun distance, $\Gamma_{\mathrm{SDS}}$ is the SD screen transmission function and $\Delta_{\mathrm{SD}}$ is the time-dependent SD degradation factor. In both Equations 4 and 5, the $d n$ values are corrected using Equation 3 in order to produce the equivalent L1B type of calibrated data from L1A uncalibrated data and OBC data. The crosstalk corrections are applied before the temperature correction.

For this work, we will focus our analysis to a selected time period in the mission. In this case, we chose data from the daytime portion of 4 orbits on July 2, 2015, which corresponds to times between UTC 09:50 and 15:25, and covers a wide variety of land, water, clear, and cloudy EV scenes. Since the coefficient values do not change significantly over time, it is expected that the contamination levels are similarly consistent. However, a study of the full mission impact of the correction to these bands will be required in the future.

Using the 4 orbits of data, we are able to analyze the impact of the correction over a wide range of scenes which includes low radiance scenes over water up to high radiance scenes over desert. To do this, we will take the radiance difference between nearest neighbor pixels and group the pixel differences into bins at their corresponding average radiance levels. While actual scene variations can cause rapid changes between neighboring pixels, on average over a large amount of data, we should measure no difference between the 2 subframes. Before applying the new correction, we measure subsample differences using this method that increase in magnitude at higher radiance levels, as seen in Figure 6 (left). The increase in the difference is nearly linear for each detector up to certain radiance level. At the highest radiance levels, there are fewer data points in each bin, and the behavior can deviate from the linear increase for some detectors.

After the new correction is applied, the subsample difference is reduced considerably over the entire radiance range. The difference is still non-zero however, and also exhibits the same linear behavior as a function of 

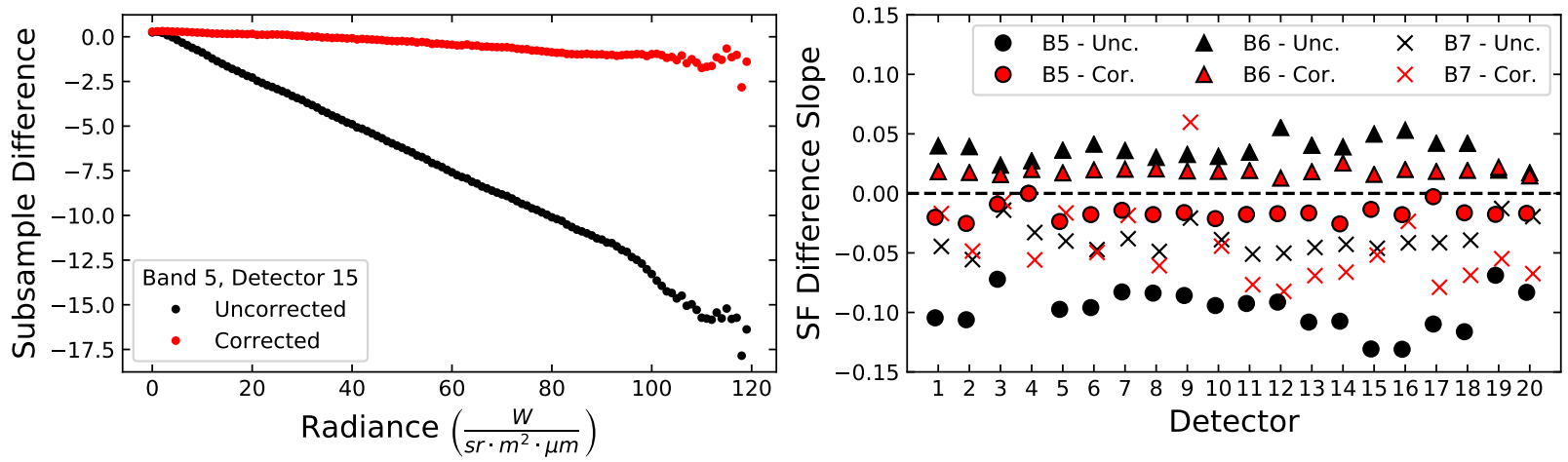

Figure 6. (Left) Example plot of the subsample difference as a function of radiance for band 5 detector 15 . The data is binned and averaged over the daytime portion of 4 orbits on July 2, 2015. (Right) The measured slope of the subsample difference for each detector in bands $5-7$ before and after the new correction is applied. For band 5 detector 4 , a value of zero is given because it is listed as inoperable in the current L1B data.

radiance level. This is true for each detector in bands $5-7$. To compare the uncorrected and corrected data, we will use the slope of the subsample difference versus radiance in order to characterize the effectiveness of the correction. A slope that is close to zero would imply a better correction to the subsample difference. While there is a small offset in the data at low radiance, this offset is negligible compared to the overall size of the subsample difference. A plot of the slope for each detector in bands $5-7$ can be seen in Figure 6 (right). In general, the impact is largest for band 5 , where the subsample difference before the correction is applied is on the order $10 \%$ for this data. This is reduced to approximately $2 \%$ for each detector within the band after the correction. For band 6 , the difference before correction is around $4 \%$, with the correction reducing the difference by approximately a factor of 2 .

For band 7, the impact is variable across the detectors. Also, in this case, the correction does not uniformly decrease the difference across each detector as was the case for bands 5 and 6 . As mentioned previously, the lunar data for band 7 does not give a clear reference to the sending signals, and this may contribute to the inability of the correction to produce good results for band 7. However, it was also noticed that the new OL correction produced greater detector differences for band 7 subframe 1. Since the subframe 1 data does not rely on the Moon, it may indicate a more fundamental difference in the behavior of band 7 that will need to be explored in the future. For that reason, the image correction results for band 7 will not be shown below.

The linear dependence of the subsample difference implies that the difference measured is due in large part to the $m_{1}$ parameter. This is because the scene variation of the sending bands at the different radiance levels for the SWIR bands is not necessarily the same as the TEBs that are responsible for the contamination. For instance, the reflectance over cloudy scenes would be relatively high compared to the thermal emission, which is typically low. It is the case that the subframe difference for the $m_{1}$ values is reduced after applying the correction, and it may also be the case that if the correction was performed "perfectly", then the $m_{1}$ values between the two subframes would be the same. This is because there is no change in the electronics configuration or scan-mirror side between the subframes other than the sampling of the 1-km bands. If these bands could be isolated, then in principle, the sampling should be identical between the two subframes. For bands 3 and 4, which are also at a nadir resolution of $500 \mathrm{~m}$ and show no signs of crosstalk contamination, we measure significantly lower $m_{1}$ differences between the subframes when compared to bands $5-7$. We will need to examine this more closely in the future.

The next test of the correction is for individual scenes in order to determine the impact of the correction on image quality. In general, the subframe differences are greater at higher radiance scenes for each band, as seen in Figure 6 (left). For band 5, the first example correction can be seen in Figure 7 for a scene over ice in Greenland. The relatively high radiance values over the ice have subframe differences on the order of $10 \%$, as expected from Figure 6. After the new correction is applied, the subframe differences are significantly reduced 
True Color Image

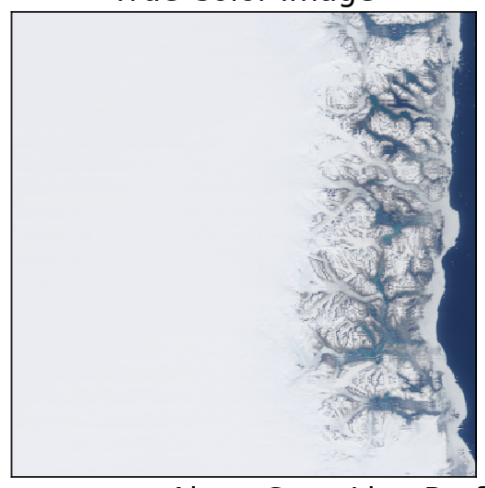

Along Scan Line Profile Comparison

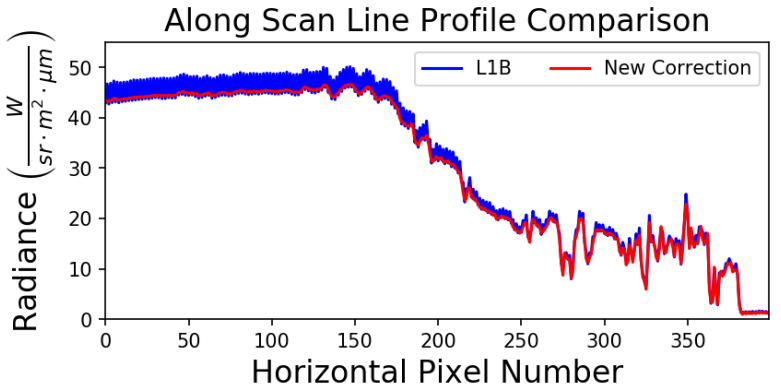

Level-1B

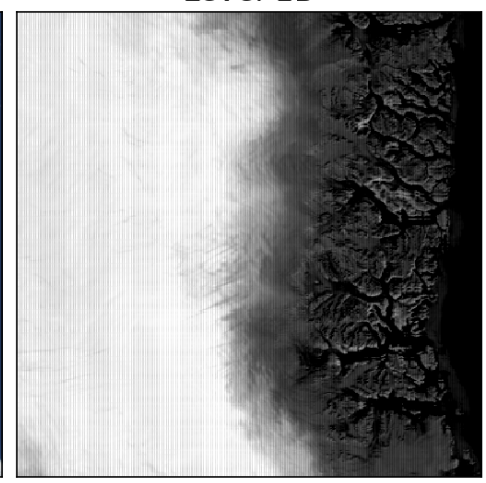

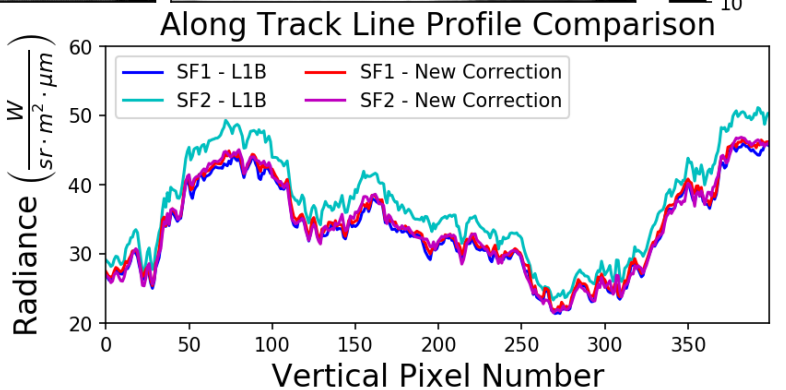

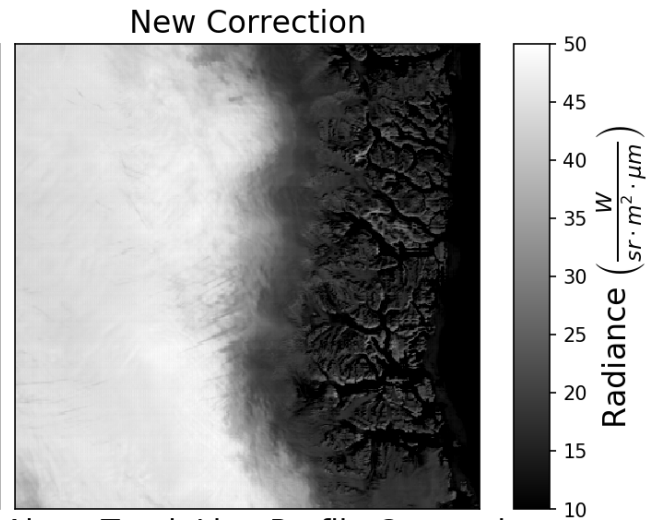

Along Track Line Profile Comparison

Figure 7. Example correction for band 5 over Greenland on July 2, 2015. (Top Left) A true color image of the scene. (Top Center) Image from the Collection 6.1 L1B product. (Top Right) Image using our new correction. For the band 5 images, data from detector 4, which is listed as inoperable in the L1B data at this time, is interpolated from detectors 3 and 5 for display purposes. (Bottom Left) Horizontal line profile through the center of the image comparing the subframe differences before and after the new correction is applied (detector 3). (Bottom Right) Vertical line profile through the center of the image showing the detector and subframe differences.

as seen in the line profiles in Figure 7. In the image comparison, we can see that the vertical striping in the image due to the subsample difference is almost entirely removed, producing a smooth image. We can also note that the correction mostly affects subframe 2, which indicates the new method for deriving the OL correction for subframe 1 in this work produces a similar result to what is currently used in the Collection 6.1 L1B.

Another example correction for band 5 is shown in Figure 8 over the Sahara Desert. This example shows a similar level of subsample difference but for a higher radiance scene. The new correction is just as effective in this case at reducing the subsample difference. Also, for this high radiance scene, the vertical line profiles also show a reduction in the detector-to-detector differences for both subframes as a result of the new correction. These detector differences are shown as a repeating spike pattern in the vertical line profile data, which are removed after the correction is applied.

For band 6 , the overall subframe differences are smaller compared to band 5 and the new correction does further reduce these differences by approximately a factor of 2 . In Figure 9, we show an example correction over the Sahara Desert. While the vertical striping that is indicative of the subsample differences is difficult to see in the images, there is a reduction in the differences that can be seen more clearly when viewing the horizontal line profile data. However, when applying the new correction, we did notice that a horizontal striping element was introduced for detector 12 of band 6 in the corrected images. This would seem to indicate an over correction for this detector using the new method even though the overall subsample difference was reduced. This issue is present for both subframe 1 and subframe 2, and might point to an issue with the derived OL correction for this detector in particular. Also, for this detector the level of contamination is noticeably higher when viewing the lunar data compared to the rest of the detectors within the band. This detector will have to be singled out for closer examination in the future. 


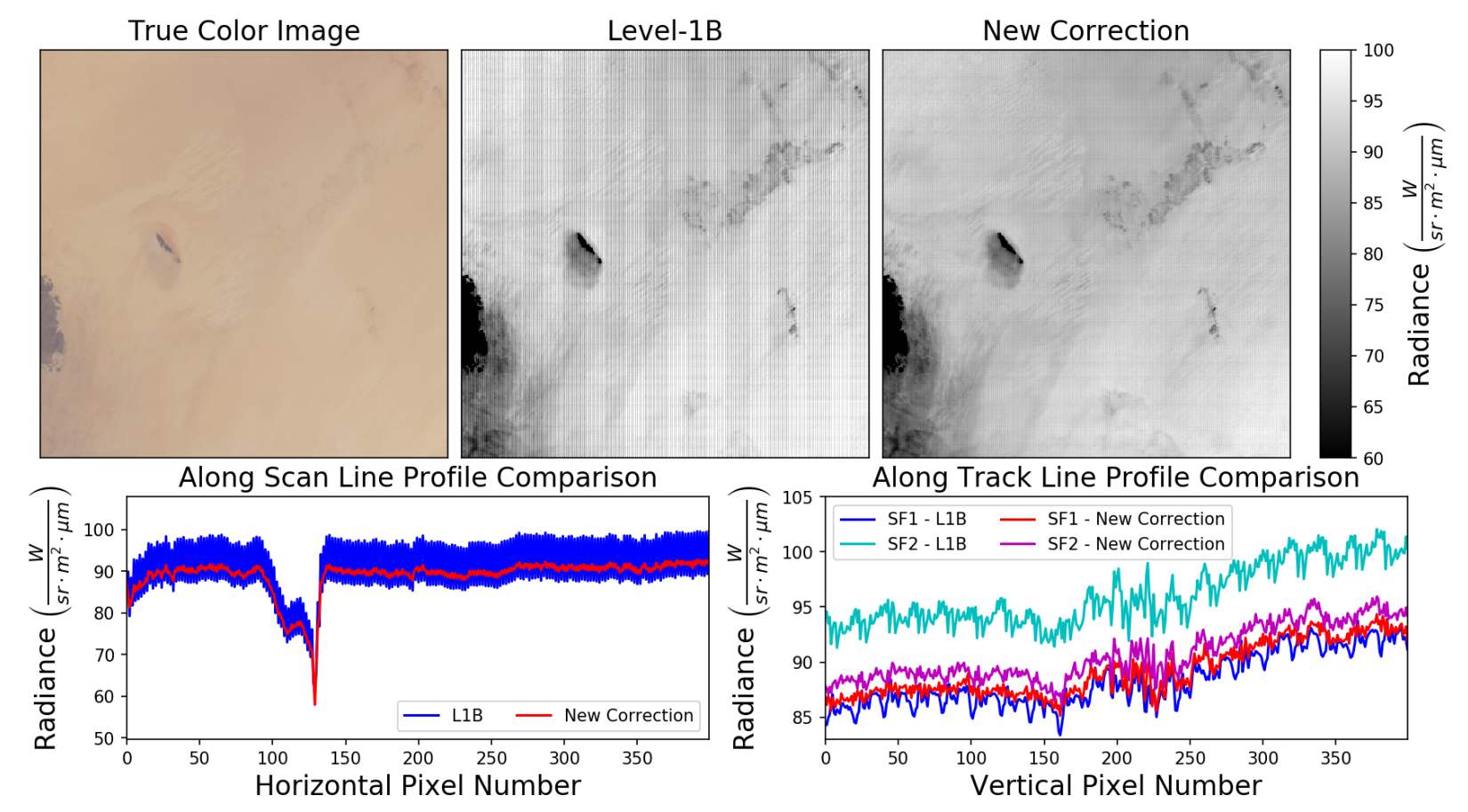

Figure 8. Example correction for band 5 over the Sahara Desert on July 2, 2015. (Top Left) A true color image of the scene. (Top Center) Image from the Collection 6.1 L1B product. (Top Right) Image using our new correction. For the band 5 images, data from detector 4, which is listed as inoperable in the L1B data at this time, is interpolated from detectors 3 and 5 for display purposes. (Bottom Left) Horizontal line profile through the center of the image comparing the subframe differences before and after the new correction is applied (detector 15). (Bottom Right) Vertical line profile through the center of the image showing the detector and subframe differences.

\section{SUMMARY AND FUTURE WORK}

In this work, we developed a new method for correcting the subsample differences observed in the 500-m SWIR bands by using sector-rotated data during lunar observations. In these observations, the EV sector was changed to view both the $\mathrm{BB}$ and the SV port, where the Moon is observed. Using the BB data, we derived a correction to the out-of-band OL signal by correlating the BB signal to data from band 25. We then derived correction coefficients from the Moon to determine electronic portion of the signal contamination. Since the sending data from the Moon saturates, we rescaled the coefficients from the Moon until the BB data was minimized. Applying these coefficients to the EV data led to a reduction in the subsample differences for bands 5 and 6 . For band 7 , the correction did not perform well, and will have to be examined in more detail.

In the future, we will further investigate the correction for band 7 in order to determine how the algorithm can be changed in order to reduce the subsample and detector differences. We will also explore the relationship between the $m_{1}$ calibration coefficient and the observed subsample differences after the correction is applied. Another possibility for improvement would be in revisiting the NTDM data with our new coefficient values. We may be able to use this data to provide additional fine adjustment parameters to the coefficient values before applying them to the $\mathrm{OBC}$ and $\mathrm{EV}$ data. Finally, we will need to investigate the long-term stability of the correction over the entire mission.

\section{ACKNOWLEDGMENTS}

The authors would like to thank Daniel Link of SSAI for his assistance in obtaining some of the instrument data. We would also like to thank Amit Angal of SSAI for his helpful comments and review of this work. 

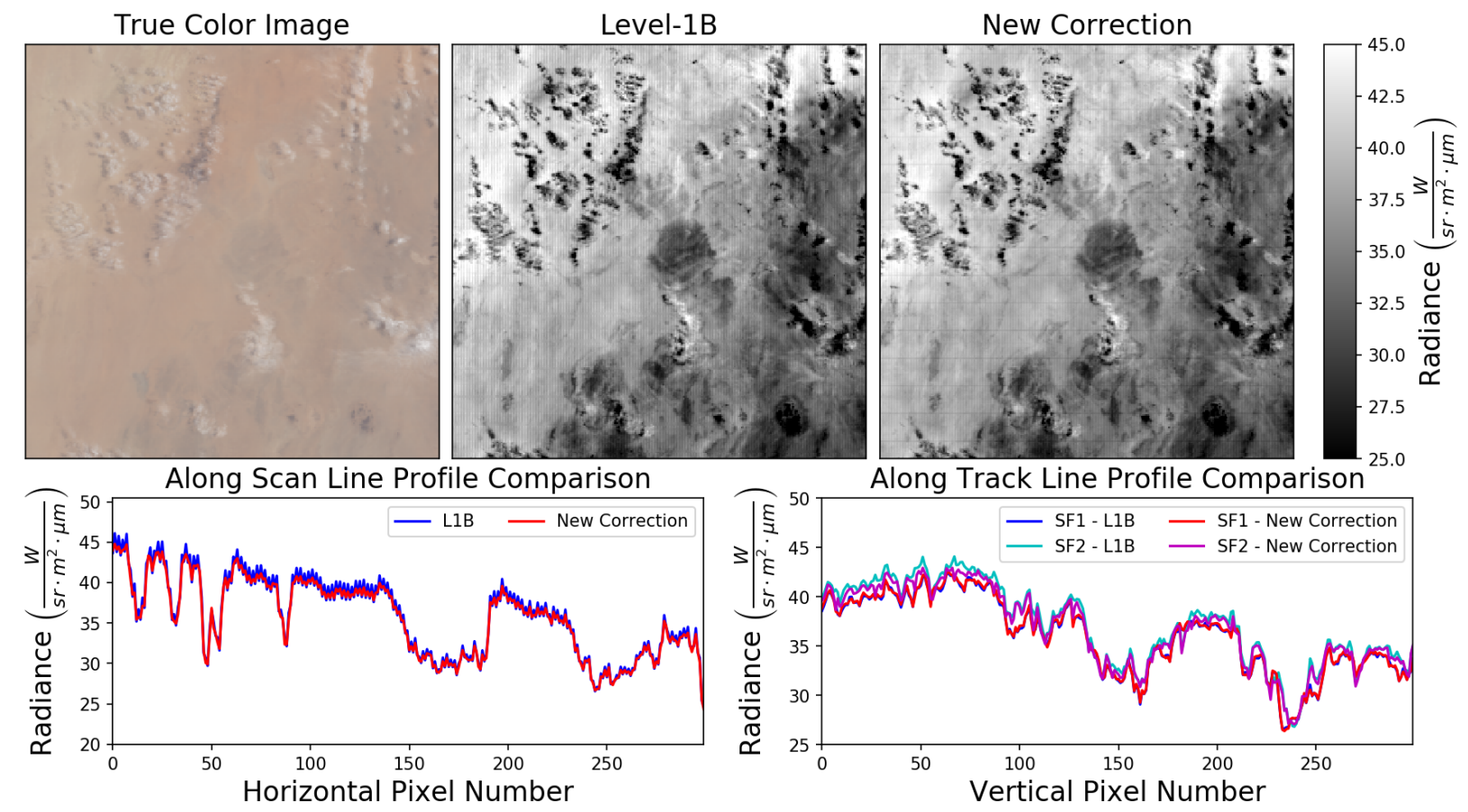

Figure 9. Example correction for band 6 over the Sahara Desert on July 2, 2015. (Top Left) A true color image of the scene. (Top Center) Image from the Collection 6.1 L1B product. (Top Right) Image using our new correction. (Bottom Left) Horizontal line profile through the center of the image comparing the subframe differences before and after the new correction is applied (detector 5). (Bottom Right) Vertical line profile through the center of the image showing the detector and subframe differences.

\section{REFERENCES}

[1] Salomonson, V. V., Barnes, W. L., Xiong, X., Kempler, S., and Masuoka, E., "An overview of Earth Observing System MODIS instrument and associated data systems performance," Proc. IGARSS 2, 1174$1176(2002)$.

[2] Xiong, X., Sun, J., Barnes, W. L., Salomonson, V. V., Esposito, J., Erives, H., and Guenther, B., "Multiyear on-orbit calibration and performance of Terra MODIS reflective solar bands," IEEE Trans. Geosci. Remote Sens. 45(4), 879 (2007).

[3] Xiong, X., Wu, A., Wenny, B. N., Madhavan, S., Wang, Z., Li, Y., Chen, N., Barnes, W. L., and Salomonson, V. V., "Terra and Aqua MODIS thermal emissive bands on-orbit calibration and performance," IEEE Trans. Geosci. Remote Sens. 53(10), 5709 (2015).

[4] Xiong, X., Che, N., and Barnes, W. L., "Terra MODIS on-orbit spatial characterization and performance," IEEE Trans. Geosci. Rem. Sens. 43(2), 355-365 (2005).

[5] Xiong, X., Che, N., and Barnes, W. L., "Terra MODIS on-orbit spectral characterization and performance," IEEE Trans. Geosci. Rem. Sens. 44(8), 2198-2206 (2006).

[6] Chen, H., Xiong, X., Angal, A., Geng, X., and Wu, A., "Alternative method of on-orbit response-versusscan-angle characterization for MODIS reflective solar bands," J. Appl. Remote Sens. 10(2), 024004 (2016).

[7] Sun, J., Xiong, X., Angal, A., Chen, H., Wu, A., and Geng, X., "Time-dependent response versus scan angle for MODIS reflective solar bands," IEEE Trans. Geosci. Remote Sens. 52(6), 3159-3174 (2014).

[8] Xiong, X., Chiang, K., Adimi, F., Li, W., Yatagai, H., and Barnes, W., "MODIS correction algorithm for out-of-band response in the short-wave IR bands," Proc. SPIE 5234, 605-613 (2004).

[9] Sun, J., Madhavan, S., Wenny, B. N., and Xiong, X., "Terra MODIS band 27 electronic crosstalk: Cause, impact, and mitigation," Proc. SPIE 8176, $81760 \mathrm{Z}$ (2011). 
[10] Sun, J., Xiong, X., Madhavan, S., and Wenny, B. N., "Terra MODIS band 27 electronic crosstalk effect and its removal," IEEE Trans. Geosci. Remote Sens. 8, 5722 (2014).

[11] Wilson, T., Shrestha, A., and Xiong, X., "Electronic crosstalk impact assessment in the Terra MODIS mid-wave infrared bands," Proc. SPIE 10423, $104231 \mathrm{Z}$ (2017).

[12] Keller, G. R., Wilson, T., Geng, X., Wu, A., Wang, Z., and Xiong, X., "Aqua MODIS electronic crosstalk survey: Mid-wave infrared bands,," IEEE Trans. Geosci. Remote Sens. (in press).

[13] Sun, J., Xiong, X., Che, N., and Angal, A., "Terra MODIS band 2 electronic crosstalk: Cause, impact, and mitigation," Proc. SPIE 7826, 78261Y (2010).

[14] Wilson, T., Wu, A., Shrestha, A., Geng, X., Wang, Z., Moeller, C., Frey, R., and Xiong, X., "Development and implementation of an electronic crosstalk correction for bands 27-30 in Terra MODIS collection 6," Remote Sens. 9(6), 569 (2017).

[15] Wilson, T., Angal, A., Shrestha, A., and Xiong, X., "Lunar calibration improvements for the short-wave infrared bands in Aqua and Terra MODIS," Proc. SPIE 10423, 1042313 (2017).

[16] Sun, J., Xiong, X., Barnes, W., and Guenther, B., "MODIS reflective solar bands on-orbit lunar calibration," IEEE Trans. Geosci. Remote Sens. 45(7), 2383 (2007).

[17] Wilson, T. and Xiong, X., "Scheduling observations of celestial objects for Earth observing sensor calibration," Proc. SPIE 10000, 1000011 (2016).

[18] Xiong, X., Sun, J., Xiong, S., and Barnes, W. L., "Using the Moon for MODIS on-orbit spatial characterization," Proc. SPIE 5234, 480 (2004).

[19] Xiong, X., Sun, J., Angal, A., Xie, Y., Choi, T., and Wang, Z., "Results of MODIS band-to-band registration characterization using on-orbit lunar observations," Proc. SPIE 8153, 81531R-1 (2011). 